\title{
Software Design of a Railway Signal Monitoring System based on Optical Fiber Sensing
}

\author{
https://doi.org/10.3991/ijoe.v14i08.9180 \\ Chun-ying $\mathrm{Ma}^{(\varpi)}$ \\ Henan Engineering Research Center of Rail Transit Intelligent Security, Zhengzhou, China \\ chunyingma283@163.com \\ Biqing Li \\ Hezhou University, Hezhou Guangxi, China
}

\begin{abstract}
The current railway track circuit monitoring system is prone to disturbances that can result in accidents. Meanwhile, basic signaling equipment is slow and cannot achieve satisfactory real-time data acquisition speed. This study aims to solve the aforementioned problems by designing an online monitoring and management platform for railway signal infrastructure, which is based on the graphical programming language LabVIEW. Online monitoring and management of railways' basic signaling equipment allow real-time collection and communication of various signal equipment data. These processes also enable signal processing, chart display, acousto-optic alarm, user authority management, data storage, data query analysis, and report printing. The test results show that the LabVIEW-based basic signaling equipment for monitoring and managing railway systems can transmit data correctly and steadily, thereby resulting in convenient and ideal operation.
\end{abstract}

Keywords - track circuit, online monitoring, LabVIEW, peak finding detection

\section{Introduction}

Railways play an important role in reducing the travel time of passengers. They promote balanced development of urban areas and rapid social and economic development. Speeding up the development of railways will not only improve the efficiency of transportation systems but also contribute to economic and social development. However, the fast development of railways has compromised traffic safety, which has become a gradually growing concern. The "7.29," "10.29," and "7.23" accidents, which devastated lives and properties, demonstrated that security problems can restrict the rapid development of railways and thus the national economy. Moreover, these accidents brought serious challenges among railway security researchers [1].

Accidents are contingent and inevitable. Recent studies have identified the main causes of several major failures of China's railways. Among these is the reliance of the existing track circuit monitoring system on the electromagnetic principle to monitor track occupancy. Because the transmission of the telecommunication number 
occurs in the open rail, it is easily interfered by lightning and other physical disturbances. Moreover, it does not guarantee redundancy in track circuit, parallel supervision, and protection mechanism. Data acquisition of existing basic signaling equipment relies on centralized collection and the relatively slow controller area network (CAN) bus transmission. This slow transmission hinders the collection of the fault's original waveform. Moreover, the existing system cannot handle real-time monitoring and analysis and other requirements for processing equipment failure.

Basic signaling equipment plays an increasingly important role in ensuring traffic safety as new railways are developed and transportation efficiency is enhanced. Improving the capacity of railways to accurately identify real-time track occupancy and latent faults is necessary. Currently, the track circuit of railways is easily affected by electromagnetic interference, and it lacks a parallel supervision guarantee mechanism. Moreover, the current basic signaling equipment has a relatively slow acquisition rate in terms of monitoring. Solving these problems will accelerate railway reform and development. This project originated from the railway signal monitoring system of Shuo Huang Railway Development Co., Ltd. and is based on optical fiber sensing.

\section{Literature review}

The currently distributed optical fiber vibration sensor system, which is based on various types of optical fiber sensing systems, has been extensively studied worldwide.

Yucel proposed a Sagnac/Mach-Zehnder interferometric distributed optical fiber sensing system that can detect phase disturbances and measure changes in phase rate. This system can also locate disturbances via the phase ratio of the demodulation after the two interference results [1]. The US Bohnert used the Sagnac fiber interferometer to Fourier transform the interference signal. By analyzing the converted points, the broadband signal was located [2]. Zhu of Fudan University applied the same principles when he modified the positioning of broadband signals, which led to the development of the Fox-Smith interferometric distributed optical fiber sensing system [3]. Meanwhile, Steindl et al. of Chongqing University used the intercorrelation algorithm to develop a bidirectional Mach-Zehnder distributed optical fiber sensing system. The phase difference of the bidirectional interference signal showed that the time delay difference was calculated, and the disturbance spot was located [4]. Sun pointed out in a research on the intelligent optical fiber sensing system that the three-dimensional vertical seismic optical fiber geophone, which was based on the Michelson optical fiber sensing system developed by Tsinghua University, could detect weak signals during oil well detection and earthquake monitoring [5]. According to Fan, the IntelliFiber distributed optical fiber vibration sensing system designed by the Israeli company Megal could identify different types of vibration signals. Moreover, it could weaken the influence of interference signals and reduce false alarm rates through adaptive signal processing [6].

Due to the simultaneous interference caused by multiple vibrations on the sensing optical fiber, distinguishing interferences from the actual positions generated by the 
vibration is impossible. Thus, Chen proposed a distributed optical fiber intrusion detection system that is based on $\phi$-OTDR. The polarized $\phi$-OTDR signal of two polarized states was obtained by installing a biased beam splitter at the receiving end. The vibration [7], meanwhile, was detected by comparing the two signals. To understand the phase information in the $\phi$-OTDR signal, Vijayan et al. divided the optical signals into equal-power signals with a three-phase difference of $120^{\circ}$ using a $3 * 3$ coupler. The phase change was derived by demodulating the blue signal using a computer [8].

The first step in signal processing of an optical fiber vibration sensor system is detecting the vibration's behavior. In practical applications, such a system must distinguish abnormal vibration signals from noise signals on time to provide an early warning. Detecting signal vibration is usually conducted using feature-based threshold comparison and model-based probability. However, because the average calculation greatly reduces the detectable response frequency, Feiner et al. developed the sliding average method and the sliding differential detection method. The sliding average method is used to reduce the random noise in the signal, whereas the sliding difference method is used to obtain detailed information about the signal. Thus, with a combination of the two techniques, the frequency response range of the system is improved [9]. Alb et al. used short-time zero crossing rate to distinguish vibration signal from low-frequency noise. This method was first applied to the end-point detection of light vibration signal, putting the corresponding improvement nest forward. However, interference signals, such as strokes and rain, are usually excluded in detecting effective vibration signals [10].

Companies that currently develop and produce signal microcomputer monitoring systems include the Beijing Whole Road Communication Signal Research and Design Institute and Ka Ke Signal Co., Ltd.

The Beijing Whole Road Communication Signal Research and Design Institute developed the TJWX-2000 signal microcomputer monitoring system, which features precise and reliable acquisition equipment designed for industrial use. This equipment's acquisition response time works in milliseconds, and its working state and signal waveform can be completely restored. In addition, the system uses highimpedance input, photoelectric conversion, electromagnetic induction, and the Holzer effect to ensure reliable electrical isolation between the acquisition and the signal safety equipment. The performance indicators thus meet the "railway signal centralized monitoring system technical conditions," as required by the Ministry of Railways [8].

Kasco Signal Co., Ltd., the leading developer and producer of domestic signal equipment, participated in the joint task force organized by the Ministry of Railways, which resulted in the development of the TJWX-97 and the TJWX-2000 type signal microcomputer monitoring systems in 1997 and 2000. Then, Kasco Signal Co., Ltd. used the original signal monitoring system to develop the TJWX-2010 signal microcomputer monitoring system, which has been applied in railway operation. In addition, Kasco Signal Co., Ltd. was tasked to write the technical conditions of the Ministry of Railways' signal microcomputer monitoring system (temporary) in 2006 and railway signal centralized monitoring system in 2010 . 
The signal microcomputer monitoring systems and monitoring products developed by other companies have similar structures and functions to those of TJWX-97 type signal microcomputer monitoring product. In other words, China's signal microcomputer monitoring system has roughly gone through the development history of the Model 97 microcomputer monitoring system, the 2000-type microcomputer monitoring system, the 2006-type microcomputer monitoring system, and the 2010-type microcomputer-based centralized monitoring system, and in essence, it doesn't have too prominent innovations.

In summary, the above research is mainly focused on the individual research of fiber-optic vibration sensing systems. However, there are few studies on the application of this technology to the monitoring of railway signals, and only relevant companies have involved them. Therefore, based on the above research status, the railway signal monitoring system based on optical fiber sensing is mainly studied.

\section{Design of detection module for optical fiber sensing track occupancy}

\subsection{Fiber Bragg grating sensing principle}

FBG, a widely used principle in the field of sensing, can be utilized to manufacture temperature and strain sensors, among others. An FBG sensor can measure various physical quantities, such as external strain and temperature. The principle of sensing is mainly about detecting the wavelength shift of the center of reflection spectrum to determine the size of the change to be measured. The reflection wavelength expression is

$$
\lambda_{B}=2 n_{e f f} \Lambda
$$

$\Lambda$ is optical fiber grating periodic parameter, and $n_{\text {eff }}$ is the effective refractive index of the fiber. When either $\Lambda$ or $n_{\text {eff }}$ is affected by external stress and temperature, the central wavelength of the fiber grating reflection spectrum will drift. Meanwhile, the relationship between the drift amount and the external measurement change is

$$
\Delta \lambda_{B} / \lambda_{B}=(1-P e) \varepsilon+(\alpha+\xi) \Delta T .
$$

The center wavelength offset of the reflection spectrum of fiber Bragg grating reflects external strain, temperature, and other measurable changes. Therefore, Bragg grating sensing can be measured by calculating the displacement of the central wavelength of the reflection spectrum. 


\subsection{Optical fiber sensing track occupancy plan design and software requirement analysis}

The principle of fiber Bragg grating sensing dictates that the track interval occupancy of the optical fiber sensing orbit is generally adopted by the method of axle counting on the basis of orbit force. For example, a set of FBG sensor counters is set at the entrance of a block and the end-point of the exit. When the log logarithm of the train wheel recorded at the entrance is equal to the logarithm of the log of the export records at the entrance, this interval does not have car occupancy. Meanwhile, when the FBG sensor is installed at the bottom of the rail, the micro strain generated by the rail will cause the central wavelength of the FBG sensor to drift as the wheel passes through the sensing point. The dynamic strain curve of the optical fiber reflection spectrum can be obtained after demodulation. Then, the fiber demodulation equipment transmits the data to the monitoring center through the Ethernet. Finally, the monitoring center calculates. Statistical analysis is used to process the strain pulse peaks to realize axle counting. An intelligent network monitor also checks the status of the occupancy/clearing of the track circuit in real time. The main object of the intelligent network monitor monitoring is the wide application of the power frequency AC continuous track circuit (480 type track circuit). The terminal voltage of the relay terminal is collected using the track circuit from the indoor signal building. The voltage value is then uploaded to the monitoring center through Ethernet. The monitoring center can identify occupancy via the working principle of the track circuit and the end voltage value of the track relay. The acquisition equipment is also used to detect the state of the occluded section of the track occupying/clearing. They form a two-totwo parallel monitoring scheme, which significantly improves the accuracy and safety of the track interval occupying/clearing state detection.

\subsection{Framework design of track occupancy detection program}

The framework of the detection program for optical fiber sensing orbit interval occupancy is designed and completed by analyzing the functions of the module and combining them with the existing framework of LabVIEW. The program framework, which consists of multi-loop synchronization, takes advantage of LabVIEW's automatic multithreading to maintain similar data collection, analysis, display, and storage. This framework has five parallel execution cycles: one event processing cycle, two TCP communication collection cycles, and two data analysis and display cycles. The relationship between the circulatory structures is shown in Figure 1.

The event handling loop responds to the front panel event. It generates commands through the front panel buttons, such as start or stop. The two collection cycles receive the acquisition commands through the queue function. Meanwhile, they receive the data packets from the optical fiber demodulation equipment and the intelligent network monitor. The notification function sends continuous read packets to the two processing display cycles. The two processing cycles show that the packets are read to the TCP separately, in accordance with the communication protocol format, algorithm analysis, result display, and data storage. The detection results of optical fiber analysis 


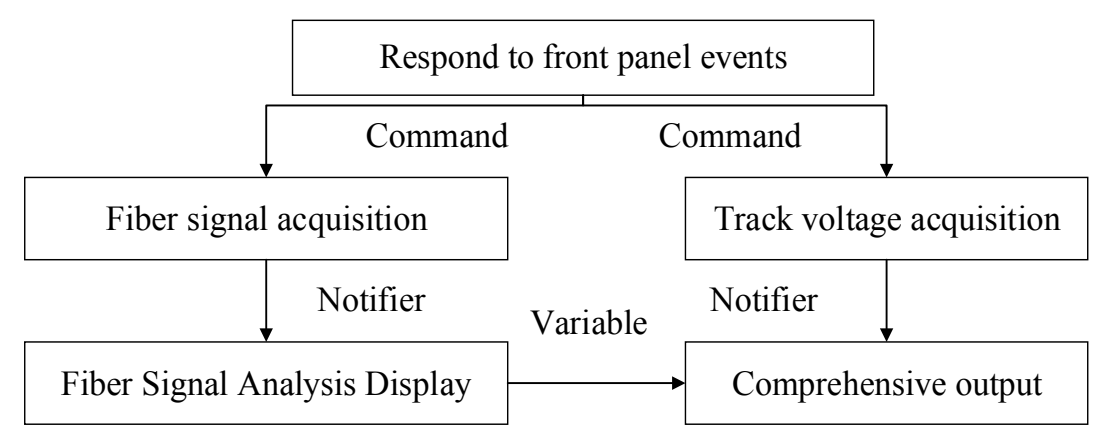

Fig. 1. Flowchart of circular structure

and processing are then passed to the track circuit by local variables. The two results are compared and synthesized into a monitoring result. Ultimately, the final track occupancy detection results are given to fulfill the purpose of "two selected, two detected."

The framework of the program is expandable. Inserting the real-time query command into the front end of the queue allows real-time data monitoring. The corresponding TCP reading and writing task is processed via the "first in, first out" principle, enabling the program framework to process data continuously. Monitoring can also prioritize realtors' requests for real-time queries.

\subsection{Fiber signal processing}

In this work, the discrete derivation method detects the maximum and minimum points of the local department. Thus, the Gauss fitting peak method needs to have an estimate of the peak height. However, different height estimates will cause a gap in calculation bandwidth, which is not suitable for real-time detection. Thus, polynomial fitting is used because of its simple algorithm, which allows for convenience. It enables small computations, noticeable effects, and extensive applications. Polynomial fitting is used after peak detection to ensure the accuracy of peak finding detection and reduce the amount of data. This process is also conducive to obtaining real-time results from the fiber grating sensing system and achieving high-accuracy detection.

Four fiber Bragg grating sensors are deemed to be within the track range if the spectral reflectance curve forms four peaks and has a total of 256 sampling points. Therefore, the whole spectral reflectance curve is cut into four parts, in accordance with the appropriate data set. Also, the Express VI fitting function is piecewise fitting. In the case of the reflection spectrum peak, the mean square and residual errors are smallest when the polynomial order selects 24 orders. The curve fitting effect near the peak is at its best.

The reflection spectrum peak formed by the optical fiber sensor is the same as the fitting principle mentioned above, all of which are fitted with the best order, according to the Express VI fitting function. When the piecewise fitting is completed, the whole fitting curve can be connected through the array insertion function of LabVIEW. The complete waveform map after polynomial fitting is shown in Figure 2. 


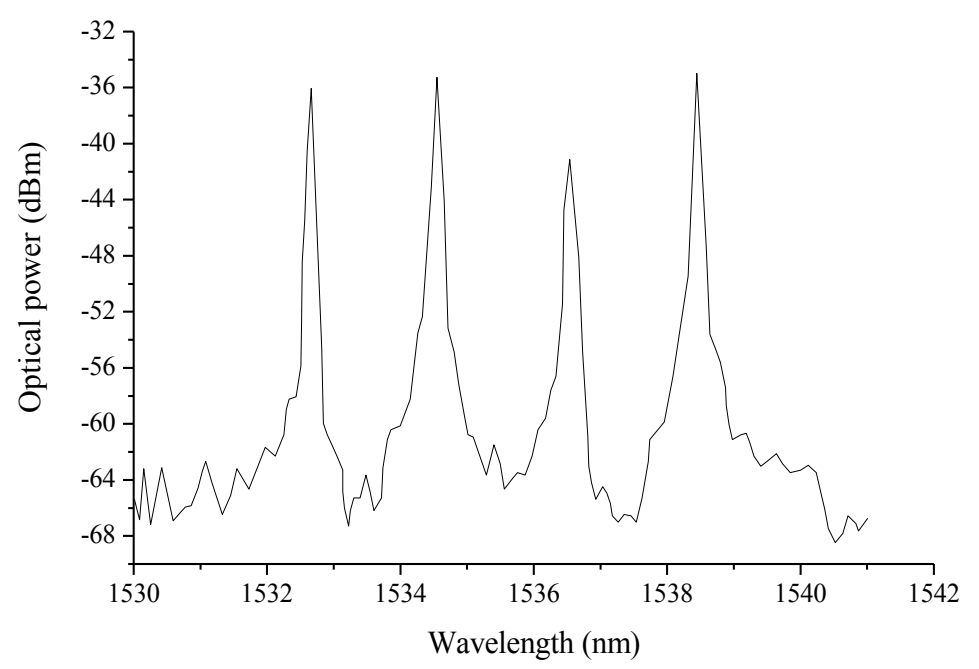

Fig. 2. Spectral signal after fitting

As shown in the FBG reflection spectrum in Figure 2, peak detection is conducted by this algorithm. The detected wave peak and fiber demodulator read the peak of the wave peak corresponding to the center wavelength, as shown in Table 1.

Table 1. Comparison between test and actual results

\begin{tabular}{|l|c|c|c|}
\hline & Actual wave peak (nm) & $\begin{array}{c}\text { Monitoring wave peak } \\
(\mathbf{n m})\end{array}$ & Error \\
\hline 1\#reflection spectrum & 1532.45 & 1532.44 & 0.01 \\
\hline 2\#reflection spectrum & 1534.51 & 1534.52 & 0.01 \\
\hline 3\#reflection spectrum & 1536.72 & 1536.74 & 0.02 \\
\hline 4\#reflection spectrum & 1538.41 & 1538.40 & 0.01 \\
\hline
\end{tabular}

As shown in Table 1, the actual wave peak position error measured by the algorithm and the optical fiber demodulator is approximately $0.01 \mathrm{~nm}$. The relative error of the detection results is nearly zero, which meets the requirements of actual measurement and control. The experimental results prove that the above-peak detection algorithm is simple and feasible. Thus, the design of the peak detection algorithm effectively improves the positioning accuracy of the central wavelength of the fiber grating sensing signal line. It also establishes a bridge between the external measurement and the discrete data sampling points of the fiber Bragg grating, which is beneficial to the subsequent axis calculation and accurate estimation of track occupancy.

\subsection{Alarm program design}

The alarm subsystem is an important part of the monitoring system. A functional alarm system with a rich interface helps monitoring personnel find, analyze, and deal 
with fault promptly. For the optical fiber sensing track occupancy detection system, the alarm is activated [11] when the results of optical fiber sensing track occupancy and track circuit monitoring are inconsistent.

When the alarm is satisfied, the system enters the alarm sub-module. First, it reads the alarm parameters from the configuration file, and then it triggers the alarm's indicator light and sound. The indicator light turns red, and the function attribute node is used to control the $500 \mathrm{~ms}$ interval of the indicator lamp once. Moreover, a voice alarm is triggered alongside the red light to alert monitoring personnel. The alarm parameter information should be stored in real time to enable the supervisor to replay the alarm information. The sound and light alarm system is automatically terminated when the fault is eliminated or when the results of the optical fiber sensing orbit and track circuit monitoring are already consistent.

\section{Design of monitoring module of switch machine and signal lamp}

\subsection{Analysis of monitoring principle and software requirements of switch machine}

The purpose of monitoring the electric switch machine via the upper computer is to reflect the resistance situation during the turnout process. It also allows on-time identification, analysis, and elimination of hidden glitches. Active power serves as the main parameter for the $\mathrm{AC}$ switch function to reflect resistance. Therefore, the monitoring contents of the PC mainly include the voltage effective value, the current effective value, the common four-wire turnout state, and the fixed anti-bit state. It can also draw the power curve and monitor the switch maneuver and the fault situation in real time.

The data acquisition control and data processing system of a host computer monitoring software has various functions. First, the computer can display the voltage, current, active power, state of common four-wire turnout, and state of the constant reverse position in the switch process of the monitoring control center. Second, the process curve of the monitoring system can be displayed in real time. Once the switch is over, all data and the original waveform of the uploaded fault are stored. Third, the supervisor can check the historical data and characteristic values from the database to each turnout conversion process. They can compare the data and analyze the display of the time curve.

\subsection{Frame design of monitoring program for switch machine}

An analysis of the functions of the data acquisition control and data processing system of the upper computer shows that the structural frame of the monitoring program of the switch machine adopts the classic program structure of LabVIEW production/consumer as the program development framework by rewriting and extending it depending on the actual situation. 
The program framework mainly consists of two cycles of TCP producers and TCP consumers. The TCP producer cycle is the top-level cycle and driven by event structure, which is mainly responsible for user events in the front panel. When a user event occurs, the system completes the conversion of specific commands in the "TCP event producer" cycle according to the action of the interface. The "TCP consumer" cycle is sent to the "TCP event consumer" by "the most front-end insert queue" function. Then, the "TCP event consumer" executes the task and sends the command to the intelligent network monitor through the "TCP write function." The "TCP consumer" loop is similar to a state machine, which mainly includes a case structure that receives different commands, executes different frames, and implements functional movements on the basis of varying conditions.

\subsection{Signal lamp monitoring module}

The signal lamp of the train is the important safety equipment. The indication state of the signal lamp is the basic guarantee of railway operation safety. A fault may result in serious accidents [12]. At present, railways have mainly two kinds of signal lights: the LED signal lamp and the double-filament incandescent signal lamp. We focus on the latter, which uses double light wires to break the main filament after breaking the wire. The filament conversion relay automatically connects the auxiliary filament to the circuit through the contact point such that the auxiliary filament is lit and the signal is displayed.

In accordance with the monitoring requirements of the Ministry of Railways, the signal lamp monitoring system collects the current and voltage of the signal lights in real time. It also analyzes the monitoring data and the data storage. The results of the monitoring system and the accumulated information can be used to evaluate the safety of the signal light and provide basis for scientific maintenance.

\section{Design of database module}

\subsection{Database program framework design}

Database structure framework programming is mainly based on queue state machine, a conceptual machine that describes a system through a directed graph. The state machine uses operations to respond to external specific events. It can operate on a series of events and can be converted through the corresponding conditions between various states. This machine mainly consists of three elements: state, event, and action.

This work aims to write three corresponding data queries VI for the signal base equipment and then embed the VI into the database operating program framework. Thus, the data can be effectively operated and dispatched. The database operation flow diagram is shown in Figure 3. 


\subsection{Data query analysis}

In view of the characteristics of multi-signal equipment and complex data types in this work, the module designs different database query subroutine and display interface. The following introduces the query and interface designs of various signal equipment and monitoring data.

Optical fiber sensing track occupancy data query is based on the starting and cutoff times to query. It queries a certain period of time occupied/cleared records of the interval. Suppose that the query time segment is the track interval occupancy/ clearing record of 2017/12/26 15:10:06 to 2017/12/26 15:20:20. In the start and time controls, 2017/12/26 15:10:06 and 2017/12/26 15:20:20 are inputted. After the "query" button is clicked, the program generates the SQL query command automatically. The query interface is shown in Figure 4.

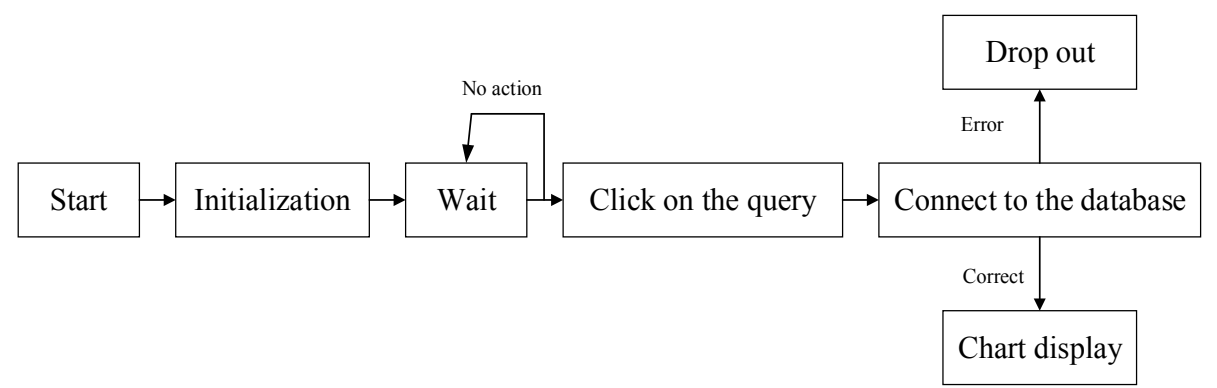

Fig. 3. Database operation flow diagram

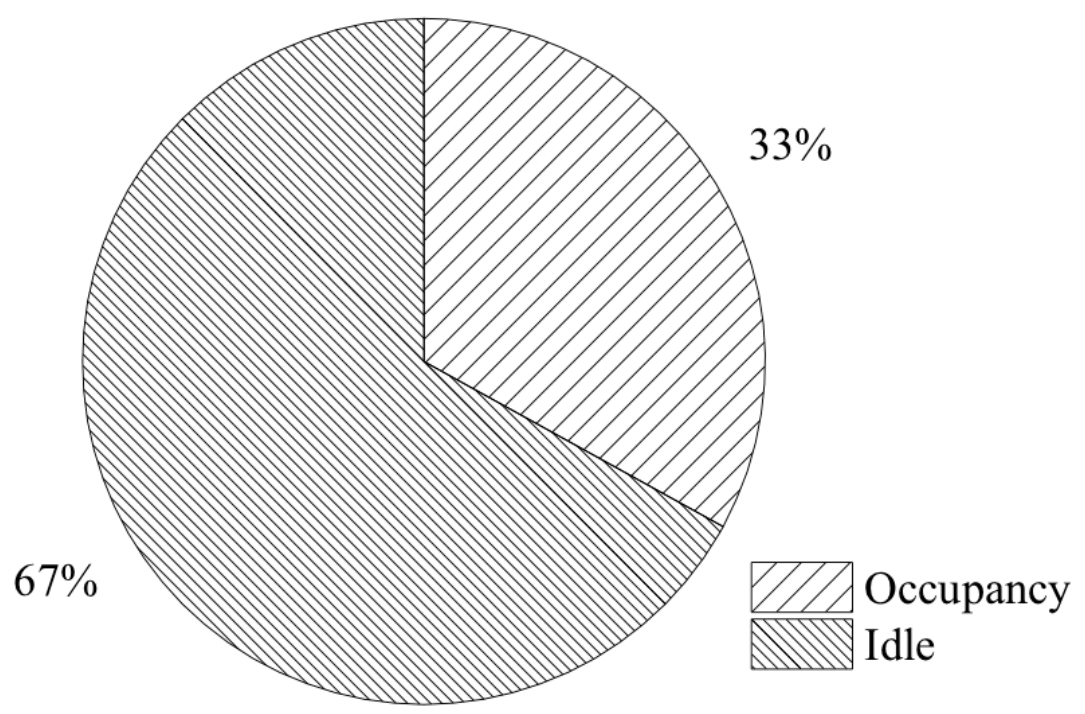

Fig. 4. Query interface 
The data query of the switch machine is mainly a daily query. The action curve and data parameters of the switch machine can be searched by inputting a specific date in the query time control. All the monitoring results are obtained during the transition process, and then the test report can be printed. For example, when you select 2017/10/24 within the query time control and click the "query" button, the program will automatically generate the SQL query command. The query interface is shown in Figure 5.

The signal data query is based on the starting and cut-off times to query, and it queries a certain period of time of the signal lights all monitoring data. For example, in checking all monitoring records from 2018/02/26 16:12:09 to 2018/02/26 16:12:20, 2018/02/26 16:12:09 and 2018/02/26 16:12:20 are entered into the start and time controls. The SQL query command is automatically generated by clicking the "query" button. The query interface is shown in Figures 6 and 7.

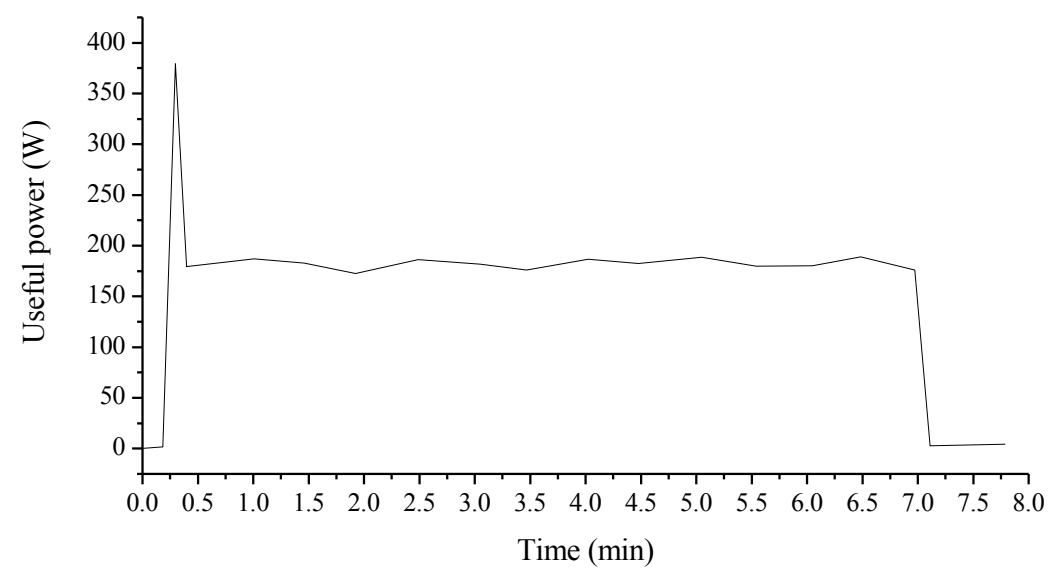

Fig. 5. Data query interface for switch machine

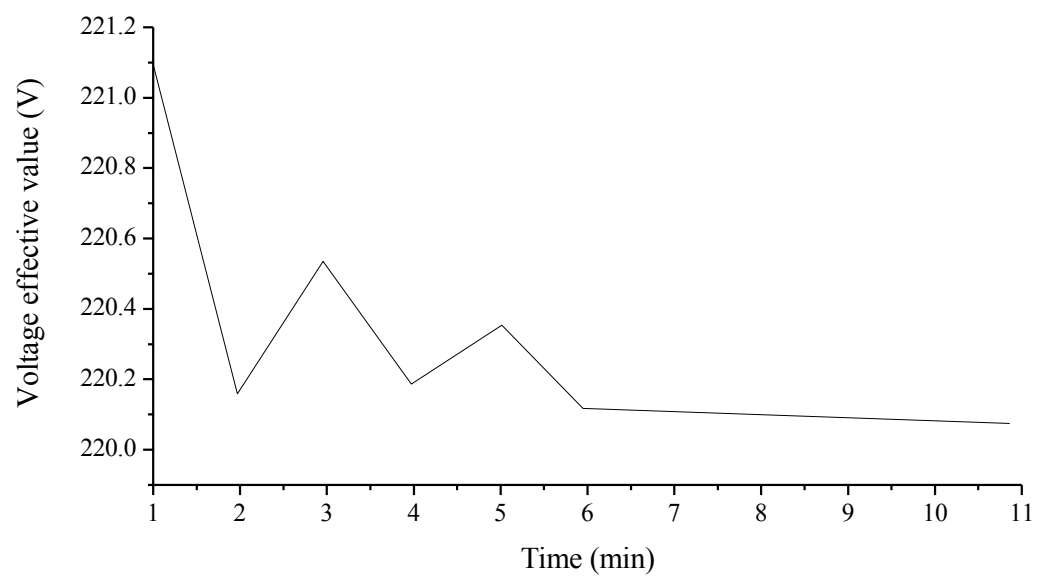

Fig. 6. Signal light data query interface (a) 


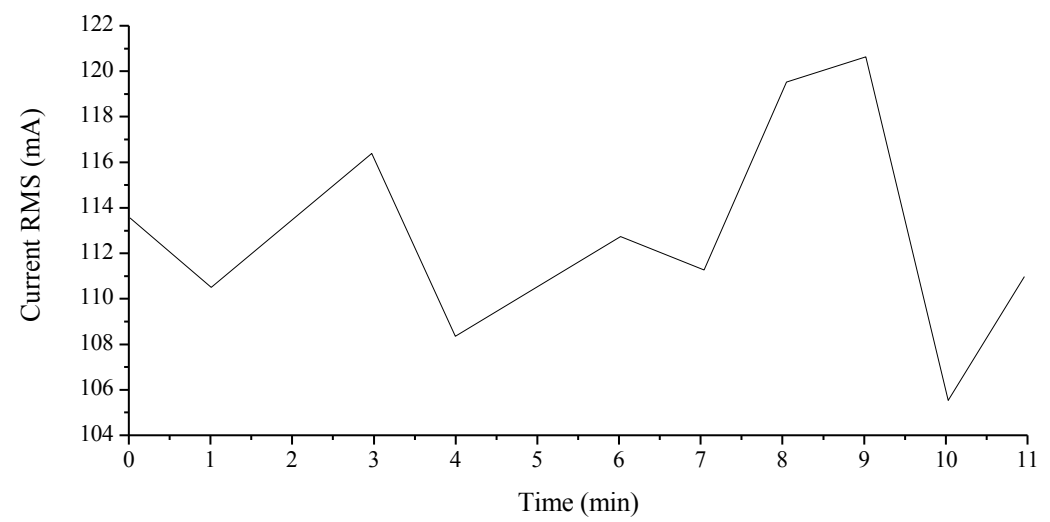

Fig. 7. Signal light data query interface (b)

\section{Conclusion}

Based on optical fiber sensing technology, the design scheme of railway signal monitoring systems is proposed. An online monitoring and management platform for railways' basic signaling equipment based on LabVIEW is designed from the point of view of an upper computer software. This design completes the dynamic monitoring interface of track occupancy detection, switch machine monitoring, signal lamp monitoring, and database inquiry and analysis. The monitoring equipment is divided into modules to facilitate unified scheduling management. The software function of optical fiber sensing track occupancy detection is completed, including the design of program frame construction, TCP/IP communication, data analysis and processing, front panel interface display alarm, and other functions. This research, particularly the design of the peak detection algorithm, lays a solid foundation for the accurate realization of the axle counting function. The whole structure and monitoring principle of the switch machine and the signal lamp monitoring system are analyzed in detail. The design of the monitoring frame is completed from the point of view of software program design. Moreover, the parts of the software modules, such as the program frame design, the data reduction analysis, the human-computer interaction interface design, and the alarm condition control. It designs the database operation module and implements data query and display.

\section{$7 \quad$ References}

[1] Yucel, M., \& Ozturk, N. F. Real-time monitoring of railroad track tension using a fiber bragg grating-based strain sensor. Instrumentation Science \& Technology, 2017, vol. (2), pp. 1-15.

[2] Bohnert, K., Hsu, C. P., Yang, L., Frank, A., Mueller, G., \& Gabus, P. Fiber-optic current sensor tolerant to imperfections of polarization-maintaining fiber connectors. Journal of Lightwave Technology, 2018, vol. 99, pp. 1-1. https://doi.org/10.1109/JLT.2018.2803807 
Paper-Software Design of a Railway Signal Monitoring System based on Optical Fiber Sensing

[3] Zhu, R., Zhang, X., Shen, D., \& Zhang, Y. Ultra broadband predistortion circuit for radioover-fiber transmission systems. Journal of Lightwave Technology, 2016, vol. 34(22), pp. 5137-5145. https://doi.org/10.1109/JLT.2016.2604395

[4] Steindl, B., Hofbauer, M., Schneider-Hornstein, K., Brandl, P., \& Zimmermann, H. Single photon avalanche photodiode based fiber optic receiver for up to $200 \mathrm{mbit} / \mathrm{s}$. IEEE Journal of Selected Topics in Quantum Electronics, 2017, vol. 99, pp. 1-1.

[5] Sun, Q., Hu, F., \& Hao, Q. Human movement modeling and activity perception based on fiber-optic sensing system. IEEE Transactions on Human-Machine Systems, 2017, vol. 44(6), pp. 743-754. https://doi.org/10.1109/THMS.2014.2354046

[6] Fan, X., Yang, G., Wang, S., Liu, Q., \& He, Z. Distributed fiber-optic vibration sensing based on phase extraction from optical reflectometry. Journal of Lightwave Technology, 2017, vol. 35(16), pp. 3281-3288. https://doi.org/10.1109/JLT.2016.2604859

[7] Chen, Z., Hefferman, G., \& Wei, T. Terahertz-range weak reflection fiber optic structures for sensing applications. IEEE Journal of Selected Topics in Quantum Electronics, 2017, vol. 99, pp. 1-1. https://doi.org/10.1109/JSTQE.2016.2612221

[8] Vijayan, A., Koneru, A., Kiamehr, S., Chakrabarty, K., \& Tahoori, M. B. Fine-grained aging-induced delay prediction based on the monitoring of run-time stress. IEEE Transactions on Computer-Aided Design of Integrated Circuits and Systems, 2018, vol. 37(5), pp. 1064-1075. https://doi.org/10.1109/TCAD.2016.2620903

[9] Feiner, R., Engel, L., Fleischer, S., Malki, M., Gal, I., \& Shapira, A., et al. Engineered hybrid cardiac patches with multifunctional electronics for online monitoring and regulation of tissue function. Nature Materials, 2016, vol. 15(6), pp. 679-685. https://doi.org/10.1038/nmat4590

[10] Alb, A. M., \& Reed, W. F. Online monitoring of molecular weight and other characteristics during semibatch emulsion polymerization under monomer starved and flooded conditions. Macromolecules, 2016, vol. 42(21), pp. 8093-8101. https://doi.org/10.1021/ma90 $\underline{1434 \mathrm{w}}$

\section{Author}

Chun-ying Ma, is with the Henan Engineering Research Center of Rail Transit Intelligent Security, Zhengzhou 451460, China. His research interests include track circuit.

Biqing Li, is a lecturer at the School of Information and Communication Engineering, Hezhou University, Hezhou Guangxi 542899, China. His research interests include Railway Signal Monitoring System.

Article submitted 15 June 2018. Resubmitted 23 July 2018. Final acceptance 03 August 2018. Final version published as submitted by the authors. 